Original article

\title{
Resilience in women with autoimmune rheumatic diseases
}

\author{
Manuel Rojas, Yhojan Rodriguez, Yovana Pacheco, Elizabeth Zapata, Diana M. Monsalve, \\ Rubén D. Mantilla, Monica Rodríguez-Jimenez, Carolina Ramírez-Santana, \\ Nicolás Molano-González, Juan-Manuel Anaya*
}

Center for Autoimmune Diseases Research (CREA), School of Medicine and Health Sciences, Universidad del Rosario, 24 No. 63 C-69 Carrera, Bogotá, Colombia

\section{A R T I C L E I N F O}

\section{Article history:}

Accepted 13 December 2017

Available online 28 December 2017

\section{Keywords:}

Resilience

Rheumatoid arthritis

Systemic lupus erythematosus

Systemic sclerosis

Sjögren's syndrome

Autoimmune Diseases

\begin{abstract}
A B S T R A C T
Objective: To evaluate the relationship between resilience and clinical outcomes in patients with autoimmune rheumatic diseases.

Methods: Focus groups, individual interviews, and chart reviews were done to collect data on 188 women with autoimmune rheumatic diseases, namely rheumatoid arthritis $(n=51)$, systemic lupus erythematosus $(n=70)$, systemic sclerosis $(n=35)$, and Sjögren's syndrome $(n=32)$. Demographic, clinical, and laboratory variables were assessed including disease activity by patient reported outcomes. Resilience was evaluated by using the Brief Resilience Scale. Bivariate, multiple linear regression, and classification and regression trees were used to analyse data.

Results: Resilience was influenced by age, duration of disease, and socioeconomic status. Lower resilience scores were observed in younger patients (<48 years) with systemic lupus erythematosus, rheumatoid arthritis, and systemic sclerosis who had low socioeconomic status, whereas older patients ( $>50$ years) had higher resilience scores regardless of socioeconomic status. There was no influence of disease activity on resilience. A particular behaviour was observed in systemic sclerosis in which patients with high socioeconomic status and regular physical activity had higher resilience scores.

Conclusion: Resilience in patients with autoimmune rheumatic diseases is a continuum process influenced by age and socioeconomic status. The ways in which these variables along with exercise influence resilience deserve further investigation.
\end{abstract}

(c) 2017 Société française de rhumatologie. Published by Elsevier Masson SAS. All rights reserved.

\section{Introduction}

Resilience is a concept that arose in the field of psychology on the second half of the 20th century. In the nineties, it became one of the main branches of research in positive psychology [1]. This concept refers to the individual's ability to recover his own homeostasis and avoid the development of a psychopathological outcome after acute or chronic stress exposure [1-3]. Thus, resilience could be considered one of the most important traits in patients suffering from chronic diseases such as autoimmune rheumatic diseases (ARDs). In ARDs, disease activity, compromised health and deterioration in functional capacity add a high charge of stress to the individual [4]. Therefore, resilience in these groups of patients could play a determining role in the outcome of the disease because the greater the

\footnotetext{
* Corresponding author.

E-mail address: juan.anaya@urosario.edu.co (J.-M. Anaya).
}

patient's resilience, the lower the vulnerability and the deleterious effects of the condition $[3,5]$.

Resilience depends on different characteristics or factors that can promote it such as optimism, active coping, social support, happiness, perseverance, meaningfulness, and existential aloneness [6]. Some early stress events such as corticoid administration or food deprivation in intrauterine life, early bouts of maternal separation and maternal care behaviour in postpartum, and social stress in infancy and adolescence have been related to the development of resilience [7].

In adulthood, the ability to bounce back is affected by stressful events such as chronic diseases which increase allostatic load (i.e., the physiological consequences accumulated through aging, as a result of repeated or chronic exposure to stress) and potentially result in physiological and psychological damage [8]. Resilience, depression, anxiety, bipolar disorder, schizophrenia and obsessivecompulsive disorder may be influenced by multiple systems like the hypothalamus-pituitary-adrenal (HPA) axis, autonomic system, and immune system [9]. Rheumatoid arthritis (RA) and systemic 
lupus erythematosus (SLE) are two major ARDs associated with psychiatric manifestations and variable resilience status [10-13]. Furthermore, patients with systemic sclerosis (SSc) and Sjögren's syndrome (SS) show high prevalence of depressive symptoms [14-16]. However, data about resilience in these ARDs are scarce. Thus, we aimed to evaluate the relationship between resilience and the clinical characteristics of RA, SLE, SSc, and SS patients.

\section{Methods}

\subsection{Study population}

A cross-sectional analytical study was performed on 188 Colombian patients with RA $(n=51)$, SLE $(n=70)$, SSc $(n=35)$, and SS $(n=32)$. The subjects have been followed in a cohort at the Center for Autoimmune Diseases Research (CREA) in Bogota, Colombia. The patients fulfilled either the 1987 American College of Rheumatology (ACR) classification criteria for RA [17], the 1997 ACR criteria for SLE [18], the 2013 ACR/European League Against Rheumatism classification criteria for SSc [19], or the revised American-European Consensus Group for SS [20]. This study was carried out in compliance with the Act 008430/1993 of the Ministry of Health of the Republic of Colombia, which classified it as minimal-risk research. The institutional review board of the Universidad del Rosario approved the study design.

\subsection{Linguistic validation of the patient-reported outcome questionnaires}

Two English proficient physicians translated the following English-original patient-reported outcome questionnaires into Spanish: "Systemic Lupus Activity Questionnaire" (SLAQ) [21], "Scleroderma Skin Patient Report Outcome" (SSPRO) [22], and the "European League against Rheumatism Sjögren Syndrome Patient Reported Index" (ESSPRI) [23]. Initially, the two physicians worked independently. Afterwards, they consolidated a single Spanish version. A mother tongue professional translator back-translated this version into English. Lastly, the physicians compared both versions to produce a second and final Spanish version. In the case of disagreement, a third English-proficient physician decided on the best version. Finally, a definite Spanish form was answered by the patients (Appendix A, Doc. S1; See the supplementary material associated with this article online).

\subsection{Data collection}

Data were obtained during individual interviews, focus groups, and chart reviews. The focus group strategy consist in a group of people (i.e., patients) brought together by a researcher to interact with them [24]. The focus group is one of the most useful strategies in clinical research, and it is applied routinely at the CREA. After patients were gathered in the same place and at the same time, a questionnaire which includes patients' socio-demographic variables such as age, age at onset, duration of disease, years of education, occupation, exercise (discrete binomial variable considered positive at least 30 minutes three times per week), and cumulative clinical data of severe clinical outcomes, including resilience, was solved by the patients. A group of expert physicians were available to perform the individual interviews if patients had doubt about the survey questions. Recording of the interviews was not done.

Clinical data included lupus nephritis in SLE, extra-articular manifestations in RA (i.e., rheumatoid nodules, lung and heart involvement, scleritis and/or episcleritis, vasculitis, Felty's syndrome, and Raynaud's phenomenon) [25], extra-glandular manifestations in SS (i.e., arthralgia, arthritis, myalgia, urticaria, photosensibility, Raynaud's phenomenon, vasculitis, dysphagia, gastritis, renal compromise, pulmonary and nervous system involvement) [26], and localized or diffuse SSc.

Disease activity was measured by the Routine Assessment of Patient Index Data 3 (RAPID3) [27], SLAQ [21], SSPRO [22], and ESSPRI [23] for RA, SLE, SSc, and SS respectively. Resilience was measured by the Brief Resilience Scale (BRS). A Spanish validated version of BRS questionnaire was used [28] (Appendix A. Doc. S1). Scoring was done according to the author's guidelines. BRS scores between 1 and 2.99 were considered low resilience; between 3 and 4.3, normal resilience; and between 4.31 and 6, high resilience [29]. The socio-economic status (SES) was categorized on the basis of national legislation and was divided into low (1 and 2), intermediate (3), and high (4 and 6) economic status as previously reported [30]. All data were collected in a secure, electronic database.

\subsection{Laboratory measurements}

Serum samples were obtained in a state of fasting. A total of 14 autoantibodies were evaluated. Detection of IgM rheumatoid factor (RF), IgG anti-CCP third-generation (CCP3), IgM and IgG anticardiolipin antibodies (ACA), IgM and IgG anti- $\beta 2$ glycoprotein-1 ( $\beta 2 \mathrm{GP1}$ ) antibodies, IgG anti-double-stranded DNA (dsDNA) antibodies, IgG thyroperoxidase (TPO) and thyroglobulin (Tg) antibodies, anti-SSA/Ro, anti-SSB/La, anti-ribonucleoprotein antibody (RNP), and anti-Smith (Sm) antibodies was done by EnzymeLinked-Immunosorbent Assay (ELISA) as previously reported [30]. Antinuclear antibodies (ANAs) were evaluated by indirect immunofluorescence assay. Serum reactivity at a dilution of at least 1/80 was considered a positive result for ANA.

\subsection{Statistical analyses}

In the univariate analysis, categorical variables were analysed by frequencies, and quantitative continuous variables were expressed as mean and standard deviation (SD) and as median and interquartile range (IQR). To assess associations between outcomes of interest and other variables, the Chi square and Kruskall-Wallis tests were used. Correlations between continuous variables were assessed by the Spearman correlation test. Multiple linear regression and classification and regression trees (CART) were used to evaluate the relationship between BRS and disease, while adjusting by duration of disease, age, and SES. Briefly, CART is a nonparametric approach in which a series of recursive subdivisions separate the data by dichotomization. The aim is to identify, at each partition step, the best predictive variable and its best corresponding splitting value while optimizing a statistical criterion. The significance level of the study was set to 0.05 . Statistical analyses were done using $\mathrm{R}$ software version 3.3.2.

\subsection{Role of the funding source}

This work was supported by Universidad del Rosario (ABN011) and Colciencias (Grant No 122254531722/Grant No0425-2013), Bogota, Colombia.

\section{Results}

\subsection{Cohort}

The characteristics of the four groups of patients with ARDs are shown in Table 1 . As expected, a lower age and early age at onset was observed in SLE patients, whereas late-onset disease was observed in SS (Table 1). SSc patients showed a shorter disease duration compared to the other ARD patients studied (Table 1). 
Table 1

General characteristics and resilience of women with autoimmune rheumatic diseases.

\begin{tabular}{|c|c|c|c|c|c|}
\hline Variable & RA & SLE & SSc & SS & $P$ value \\
\hline \multicolumn{6}{|l|}{ Sociodemographic data } \\
\hline Age (IQR) & $58(48.5-63)$ & $50.5(37.5-57)$ & $58(51.5-62.5)$ & $64.5(55.7-68.7)$ & $<0.001$ \\
\hline Age at onset disease (IQR) & $36(26-49)$ & $29(22-40)$ & $48(37-53.5)$ & $50.5(40-58.25)$ & $<0.001$ \\
\hline Disease duration (IQR) & $17(10.5-26)$ & $13(9-21.75)$ & $7(4-13)$ & $12(9-17)$ & $<0.01$ \\
\hline Educational years (IQR) & $14(8-17)$ & $14(11-16)$ & $11(9-16)$ & $13.5(11-16)$ & 0.78 \\
\hline Socioeconomic status (\%) & & & & & $<0.01$ \\
\hline Low & $18(36)$ & $19(27.1)$ & $8(24.2)$ & $3(9.4)$ & \\
\hline Middle & $14(28)$ & $39(55.7)$ & $15(45.5)$ & $15(46.9)$ & \\
\hline High & $18(36)$ & $12(17.14)$ & $10(30.3)$ & $14(43.7)$ & \\
\hline ND & $1(1.9)$ & & $2(5.7)$ & & \\
\hline Occupation (\%) & & & & & $<0.01$ \\
\hline Employed & $30(59)$ & $44(63)$ & $20(57)$ & $11(34)$ & \\
\hline Unemployed & $20(39)$ & $26(37)$ & $15(43)$ & $20(63)$ & \\
\hline ND & $1(1.9)$ & & & $1(3)$ & \\
\hline Exercise (\%) & $22(43.1)$ & $28(40)$ & $23(65.7)$ & $17(53.1)$ & 0.071 \\
\hline \multicolumn{6}{|l|}{ Resilience assessment } \\
\hline Total BRS (IQR) ${ }^{\mathrm{b}}$ & $3.33(3.1-4.1)$ & $3.42(3-3.83)$ & $3.33(2.8-3.7)$ & $3.25(2.95-4)$ & 0.5 \\
\hline $\operatorname{Low}(\%)^{\mathrm{c}}$ & $8(15.7)$ & $11(15.7)$ & $10(28.6)$ & $8(25)$ & 0.5 \\
\hline Normal (\%) ${ }^{\mathrm{d}}$ & $32(62.7)$ & $50(71.4)$ & $21(60)$ & $20(62.5)$ & 0.5 \\
\hline $\operatorname{High}(\%)^{\mathrm{e}}$ & $11(21.6)$ & $9(12.9)$ & $4(11.4)$ & $4(12.5)$ & 0.5 \\
\hline
\end{tabular}

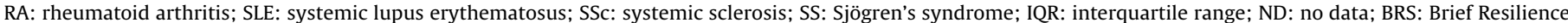
Scale Total Score (i.e., total sum by the total number of questions answers).

a At least 30 minutes three times per week.

b A multiple linear regression analysis, classification and regression trees (CART) were performed to evaluate the relationship between BRS and disease, adjusting by duration of disease, age and socioeconomic status which disclosed statistical differences (Fig. 1).

c Low BRS: 1-2.99.

d Normal BRS: $3-4.3$.

e High BRS: 4.31-6.

Table 2

Autoantibodies in women with autoimmune rheumatic diseases at the time of the study.

\begin{tabular}{lccccl}
\hline Autoantibody (\%) & RA & SLE & SSc & SS & $P$ \\
\hline ANAs & $4(7.8)$ & $59(84.3)$ & $12(34.3)$ & $16(51.6)$ & $<0.0001$ \\
SSA/Ro & $11(21.6)$ & $28(40)$ & $12(34.3)$ & $23(74.2)$ & $<0.0001$ \\
SSB/La & $3(5.9)$ & $5(7.1)$ & $6(17.1)$ & $15(48.4)$ & $<0.0001$ \\
Sm & $0(0)$ & $13(18.6)$ & $2(5.7)$ & $0(0)$ & 0.0004 \\
RNP & $2(3.9)$ & $27(38.6)$ & $6(17.1)$ & $5(16.1)$ & $<0.0001$ \\
ACA IgG & $2(3.9)$ & $13(18,6)$ & $2(5.7)$ & $1(3.2)$ & 0.0153 \\
ACA IgM & $7(13.7)$ & $11(15.7)$ & $8(22.8)$ & $2(6.5)$ & 0.3119 \\
B2GP1 IgG & $0(0)$ & $6(8.6)$ & $1(2.9)$ & $1(3.2)$ & 0.1279 \\
B2GP1 IgM & $2(4)$ & $7(10)$ & $8(22.9)$ & $3(9.7)$ & 0.0473 \\
RF & $43(84.3)$ & $24(34.3)$ & $23(65.7)$ & $20(64.5)$ & $<0,0001$ \\
CCP3 IgG & $39(76.5)$ & $1(1.4)$ & $2(5.7)$ & $1(3.2)$ & $<0,0001$ \\
dsDNA & $4(7.8)$ & $33(47.1)$ & $3(8.6)$ & $6(19.4)$ & $<0,0001$ \\
TPO Abs & $14(27.5)$ & $8(11.4)$ & $6(17.1)$ & $7(22.6)$ & 0.1471 \\
Tg Abs & $6(11.8)$ & $5(7.1)$ & $4(11.4)$ & $6(19.4)$ & 0.3558 \\
\hline
\end{tabular}

RA: rheumatoid arthritis; SLE: systemic lupus erythematosus; SSc: systemic sclerosis; SS: Sjögren's syndrome; SD: standard deviation; Abs: antibodies; ANAs: antinuclear antibodies; Sm: anti-Smith antibodies; RNP: ribonucleoprotein; ACA: anti-cardiolipin antibodies; $\beta 2 \mathrm{GP} 1$ : anti- $\beta 2$ glycoprotein-1; dsDNA: anti-doublestranded DNA; RF: rheumatoid factor; CCP3: anti-CCP third-generation; TPO: Thyroperoxidase; Tg: thyroglobulin.

a Data correspond to number of patients (\%).

Educational level and occupational status were similar among ARDs. Autoantibodies results are shown in Table 2.

\subsection{Clinical outcomes}

Extra-articular manifestations were registered in 17 (33.3\%) patients with RA. Lupus nephritis was observed in $50 \%$ of SLE patients. Only $2(5.7 \%)$ patients with SSc evinced diffuse subtype and $29(90.6 \%)$ SS patients showed extraglandular manifestations. Resilience scores were similar among the groups of patients regardless of the severity of clinical outcomes.

\subsection{Clinical status, demographic factors and resilience}

According to the RAPID3 score, $52.9 \%$ of RA patients showed high disease activity. The median SLAQ and ESSPRI scores for SLE and SS were 16 (IQR 8.25-26), and 6 (IQR 4.7-6.75) respectively. The median SSPRO score for SSc was 52 (IQR 30.5-64). The median BRS score for all ARDs was 3.33 (IQR 3-3.8) with no significant differences among groups (Table 1 ). There was no correlation between disease activity and BRS score.

Since a significant difference was observed for age, SES, and duration of disease among groups, a multiple linear regression analysis was done (Table 1). Results showed that the BRS score was significantly higher in RA than in SSc $(P=0.01)$. Furthermore, decision tree analyses revealed three patient groups (Fig. 1). RA, SLE and SSc patients under 48 years old with low SES had low resilience scores (Fig. 1BI), whereas those between 48 and 64 years old with RA and SSc had high BRS scores regardless of SES (Fig. 1BII). Patients above 50 years with SLE and SS showed high resilience levels (Fig. 1BIII) and BRS was significantly higher in this group compared to patients under 48 years old with SLE and SS (Fig. 1BI-III).

A particular behaviour was observed in SSc in which patients with higher SES and regular physical activity evinced higher resilience scores (Fig. 2). No differences in resilience scores were obtained concerning autoantibodies or treatment in all ARDs evaluated (data not shown).

\section{Discussion}

Resilience was evaluated simultaneously in four groups of Colombian patients with ARDs by the same team and in an identical manner. The results indicate resilience is influenced by age and socioeconomic status.

Chronic diseases may affect the patient's psychological status [3]. Inflammatory states and allostatic disease load have been recognized as triggering factors of psychological distress [2,13,31]. Resilience, i.e., the ability to bounce back, has been studied in chronic conditions (e.g., diabetes, RA, SLE, cancer, etc.) $[2,11,12]$. 
A.

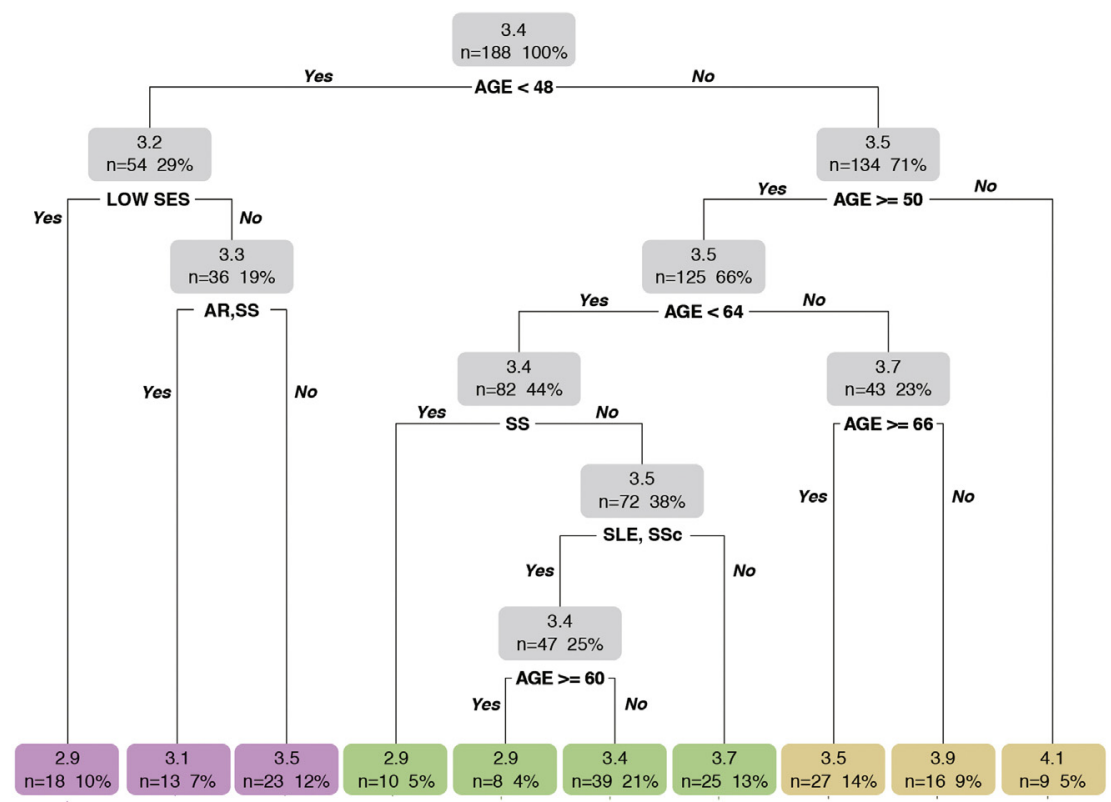

B.
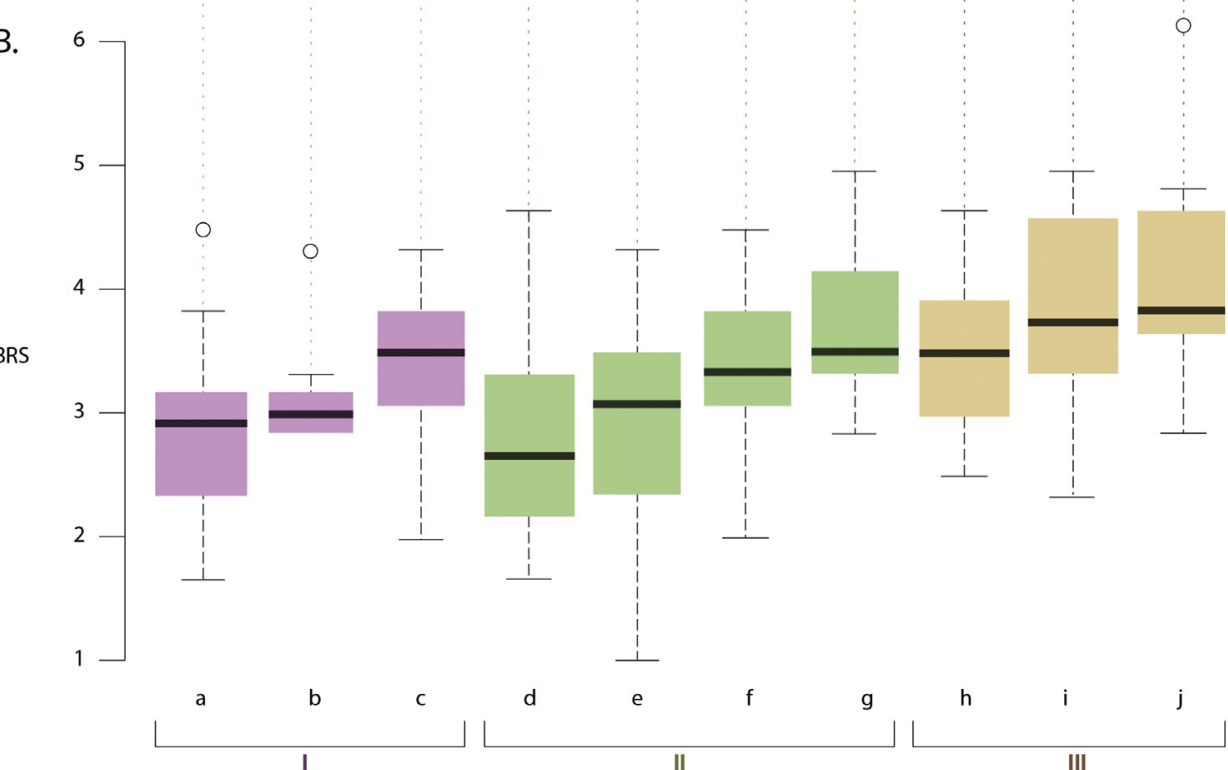

O

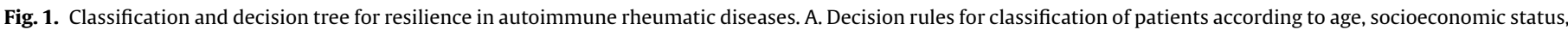

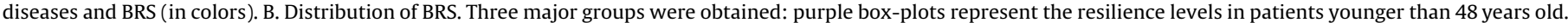

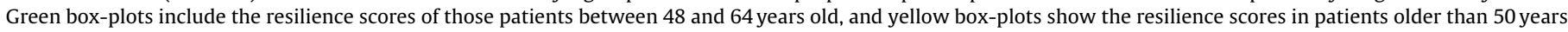

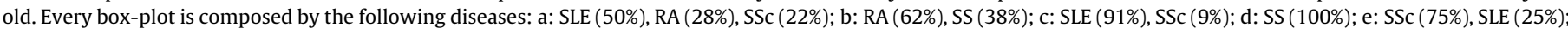

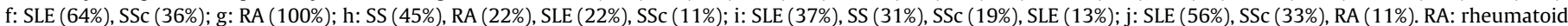

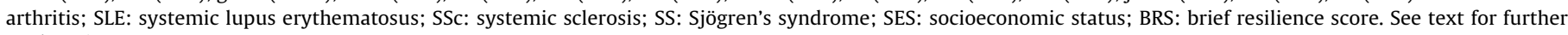
explanations.

As confirmed by our study, it is considered to be a continuous process rather than a stable personality trait [32]. Nevertheless, the use of different scales to measure it precludes comparisons between studies [33].

Conflicting evidence regarding age and the duration of disease as modifiers of resilience have emerged in SLE and RA. Cal et al. [11] applied the Wagnild and Young resilience scale and found that patients with SLE under 35 years old had the highest resilience scores. In addition, Faria et al. [10], applying the same scale found that resilience in SLE was inversely correlated with age and duration of disease. In contrast, observations of younger patients with SLE have suggested that they may perceive a limitation on their physical and social abilities which could affect resilience at an early age [34]. On the other hand, studies of resilience in RA are scarce. Li et al. [12] reported that age was not correlated with resilience. This observation is in line with our study in which neither patients' ages nor disease duration correlated with resilience. However, the decision tree analysis revealed that young patients with SLE and SSc (i.e., under 48 years old) had higher resilience levels, whereas RA and SS patients had lower ones (Fig. 1BI). Furthermore, in patients over 50 years, SLE and SS showed higher resilience scores (Fig. 1BIII) and BRS was significantly higher in this group when compared with patients under 48 years old (Fig. 1BI-III).

Although duration of disease was higher in RA patients, SLE patients appeared to build up resilience throughout the disease process, probably since diagnosis is made early in comparison with other ARDs (Table 1) (Fig. 1BIII), thus highlighting resilience as a continuum and adaptive process in SLE. In addition, since patients 

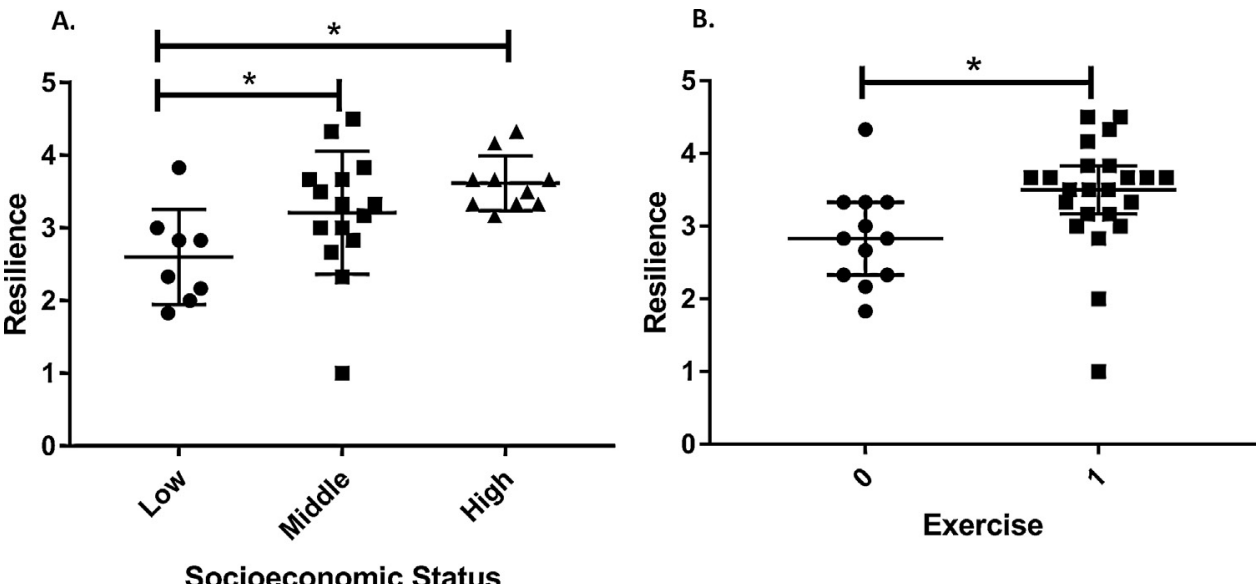

Fig. 2. Box plots of resilience in patients with systemic sclerosis by A. Socioeconomic status, and B. Exercise. ${ }^{*} P<0.05$.

with RA, SSc, and SS tended to have a later onset of disease, a higher load of lifetime experiences may favour BRS scores.

It has been suggested that gender, years of formal education, SES, and occupation influence resilience in patients with chronic diseases [2]. Women with low SES and a low level of education have had a higher incidence of psychiatric illnesses, especially after stressful life events [3]. Azizoddin et al. [35] stated that low SES was associated with higher symptoms of depression and anxiety through the effects of psychosocial resilience in SLE. These data are similar to our results since patients with low SES presenting with SLE, SSc, and SS had lower resilience scores (Fig. 1BI). Overall, these observations point out the complexity of the concept of resilience in which multiple parameters may play a role. This study has shown that disease by itself is not associated with the resilience scores, but when combined with other factors such as age and SES, the differences in the resilience rates among the different ARDs become evident.

Concerning occupational status, previous studies showed that unemployed patients with SLE did not have different resilience levels [11] and, interestingly, resilience was positively correlated with workload (i.e., working hours) [10]. In contrast, our study demonstrated no differences regarding occupational status and BRS score. Furthermore, although educational level has been related to enhanced resilience response in chronic diseases, our results did not show a correlation between resilience and years of education among patients with the four ARDs evaluated. Thus, neither educational nor occupational status appear to be related to the ability to bounce back in Colombian patients with ARDs. Whether resilience is population-specific is unknown. Gene-gene and geneenvironment interactions underlying inter-individual variability in stress responses have been evaluated [36]. Thus, the genetic factors of resilience, including ancestry, deserve further evaluation [37].

Exercise itself is a stressing phenomenon that increases the activity of the HPA axis and the sympathetic nervous system [38]. However, regular, repeated, intermittent exposure to exercise, with enough time to recover in between, can lead to physiological 'stress training', which, in the end, helps patients to respond better to psychological and physical stress [38]. In our study, it appears that SSc patients who exercise at least 30 minutes three times per week exhibited higher resilience scores than those who did not (Fig. 2). Conversely, previous reports have suggested that exercise in SSc intensifies systemic inflammation and oxidative stress [39]. However, regular physical activity in SSc has been correlated with lower symptoms of anxiety, depression, and fatigue and a higher ability to participate in social roles and activities [40]. Therefore, the suggestion that physical activity, when not excessive, plays a positive role in improving quality of life in patients with SSc deserves further confirmation.

Patients in our study did not show evidence of different resilience scores regardless of clinical outcomes (i.e., lupus nephritis in SLE, extra-articular manifestations in RA, extra-glandular manifestations in SS, and localized or diffuse SSc) or self-reported disease activity. Allostatic disease load has been recognized as a critical factor for psychiatric and chronic diseases [8], which puts a high burden on quality of life, particularly for those patients with poor social support [2]. Physical disability and social dysfunction are directly associated with maladaptation to illnesses [41]. This issue is secondary to different aspects of illness such as disease activity, systemic manifestations, comorbidities, and hospitalizations [42]. In agreement with our results, Li et al. [12] described a lack of correlation between disease activity and a resilience score in RA patients. In addition, Ostojic et al. [43] found that severity of symptoms in SSc was not associated with development of depression. It has been recognized that patients who face hardship could have learned from their experiences and may apply the acquired knowledge to coping with their illness [2]. Our results may imply that patients with ARDs facing worse clinical outcomes present better control of stress and develop an improved response to adversity. Moreover, as previously reported by studies on minority groups, factors such as spirituality and culturally relevant activities could have contributed to resilience [2]. In Colombia, $90 \%$ of the people profess the Christian faith [http://www. worldatlas.com/articles/religious-beliefs-in-colombia.html].

Although coping, social support, self-efficacy and compliance have been associated with resilience; they were not evaluated in this work. However, according to our study design, we aimed to evaluate the association among resilience, sociodemographic variables, habits (e.g., exercise) and severity of disease. Thus, the relationship between resilience and the above-mentioned characteristics deserves further investigation in ARDs.

In summary, resilience in Colombian patients with ARDs is a continuum process influenced by age, SES, and duration of disease. Interestingly, previous factors associated with resilience (e.g., occupation, education level) were not associated with it in this study, thus suggesting that these features do not play a key role in the development of resilience in our patients with ARDs. Our results could aid in setting up behavioral training programs in resilience since they have been proven to improve quality of life, pain, and self-efficacy [44]. However, since inheritable and environmental factors, including epigenetics [37], spirituality, and cultural activities may influence resilience [2], further studies that take these facets into account in ARDs are warranted. 


\section{Disclosure of interest}

The authors declare that they have no competing interest.

\section{Acknowledgments}

The authors would like to thank Janneth Sanchez-Cuervo, Janeth Perez-Cristiano, Juliana Saavedra, Julian Barahona-Correa and Diana González-Bravo for their important contributions at the beginning of this study, and Cecile Dunn for her contribution in the linguistic validation of the patient-reported outcome questionnaires.

\section{Appendix A. Supplementary data}

Supplementary data (Doc. S1) associated with this article can be found, in the online version, at https://doi.org/10.1016/ j.jbspin.2017.12.012.

\section{References}

[1] Margarita J, Vinaccia S. Resiliencia: una perspectiva desde la enfermedad crónica en población adulta. Pensam Psicológico 2011;9:69-82.

[2] Stewart DE, Yuen T. A systematic review of resilience in the physically ill. Psychosomatics 2011;52:199-209.

[3] Tosevski DL, Milovancevic MP. Stressful life events and physical health. Curr Opin Psychiatry 2006;19:184-9.

[4] Quiceno JM, Vinaccia S. Resiliencia, percepción de la enfermedad, creencia y afrontamiento espiritual religioso y calidad de vida relacionada con la salud en pacientes con diagnóstico de artritis reumatoide. Psicol Desde El Caribe 2013;30:590-619.

[5] Smith BW, Zautra AJ. Vulnerability and resilience in women with arthritis: test of a two-factor model. J Consult Clin Psychol 2008;76:799-810.

[6] Smith BW, Dalen J, Wiggins K, et al. The brief resilience scale: assessing the ability to bounce back. Int J Behav Med 2008;15:194-200.

[7] Lupien SJ, McEwen BS, Gunnar MR, et al. Effects of stress throughout the lifespan on the brain, behaviour and cognition. Nat Rev Neurosci 2009;10:434-45.

[8] McEwen BS. Stress, adaptation, and disease: allostasis and allostatic load. Ann N Y Acad Sci 1998;840:33-44.

[9] Elenkov IJ, Chrousos GP. Stress hormones, proinflammatory and antiinflammatory cytokines, and autoimmunity. Ann N Y Acad Sci 2002;966:290-303.

[10] Faria DAP, Revoredo LS, Vilar MJ, et al. Resilience and treatment adhesion in patients with systemic lupus erythematosus. Open Rheumatol J 2014;8:1-8.

[11] Cal SF, Santiago MB. Resilience in systemic lupus erythematosus. Psychol Health Med 2013;18:558-63.

[12] Liu L, Xu X, Xu N, et al. Disease activity, resilience and health-related quality of life in Chinese patients with rheumatoid arthritis: a multi-center, crosssectional study. Health Qual Life Outcomes 2017;15:1-10.

[13] Evers AWM, Zautra A, Thieme K. Stress and resilience in rheumatic diseases: a review and glimpse into the future. Nat Rev Rheumatol 2011;7:409-15.

[14] Baubet T, Ranque B, Taïeb O, et al. Mood and anxiety disorders in systemic sclerosis patients. Press Med 2011:40:111-9.

[15] Westhoff G, Dörner T, Zink A. Fatigue and depression predict physician visits and work disability in women with primary Sjögren's syndrome: results from a cohort study. Rheumatology (Oxford) 2012;51:262-9.

[16] Matsuura E, Ohta A, Kanegae F, et al. Frequency and analysis of factors closely associated with the development of depressive symptoms in patients with scleroderma. J Rheumatol 2003;30:1782-7.

[17] Kasturi S, Goldstein BL, Malspeis S, et al. Comparison of the 1987 American College of Rheumatology and the 2010 American College of Rheumatology/European League against Rheumatism Criteria for Classification of Rheumatoid Arthritis in the Nurses' Health Study Cohorts. Rheumatol Int 2014;34:407-11.

[18] Inês L, Silva C, Galindo M, et al. Classification of systemic lupus erythematosus: systemic lupus international collaborating clinics versus American College of
Rheumatology Criteria. A comparative study of 2,055 patients from a real-life, international systemic lupus erythematosus cohort. Arthritis Care Res (Hoboken) 2015;67:1180-5

[19] Van den Hoogen F, Khanna D, Fransen J, et al. 2013 classification criteria for systemic sclerosis: an American College of Rheumatology/European league against Rheumatism collaborative initiative. Arthritis Rheum 2013;65:2737-47.

[20] Goules AV, Tzioufas AG, Moutsopoulos HM. Classification criteria of Sjögren's syndrome. J Autoimmun 2014;48-49:42-5.

[21] Karlson EW, Daltroy LH, Rivest C, et al. Validation of a Systemic lupus activity questionnaire (SLAQ) for population studies. Lupus 2003;12:280-6.

[22] Man A, Correa JK, Ziemek J, et al. Development and validation of a patientreported outcome instrument for skin involvement in patients with systemic sclerosis. Ann Rheum Dis 2017;76:1374-80.

[23] Seror R, Theander E, Brun JG, et al. Validation of EULAR primary Sjögren's syndrome disease activity (ESSDAI) and patient indexes (ESSPRI). Ann Rheum Dis 2015;74:859-66.

[24] Traynor M. Focus group research. Nurs Stand 2015;29:44-8.

[25] Ortega-Hernandez O-D, Pineda-Tamayo R, Pardo AL, et al. Cardiovascular disease is associated with extra-articular manifestations in patients with rheumatoid arthritis. Clin Rheumatol 2009;28:767-75

[26] Sarmiento-Monroy JC. Espectro clinico y subfenotipos. In: Anaya J-M, Sarmiento-Monroy JC, García-Carrasco M, editors. Síndrome de Sjögren. Segunda ed, Bogotá: Editorial Universidad del Rosario; 2017. p. 20-40.

[27] Pincus T, Swearingen CJ, Bergman M, et al. RAPID3 (Routine Assessment of Patient Index Data 3), a rheumatoid arthritis index without formal joint counts for routine care: proposed severity categories compared to disease activity score and clinical disease activity index categories. J Rheumatol 2008;35:2136-47.

[28] Rodriguez-Rey R, Alonso-Tapia J, Hernansaiz-Garrido H. Reliability and validity of the Brief Resilience Scale (BRS) Spanish Version. Psychol Assess 2016;28:e101-10.

[29] Smith BW, Epstein EM, Ortiz JA et al. The foundations of resilience: what are the critical resources for bouncing back from stress? In: Prince-Embury S, Saklofske $\mathrm{DH}$, editors. Resil. Child. Adolesc. Adults Transl. Res. into Pract. Springer New York: New York, NY; 2013. p. 167-87.

[30] Franco J-S, Amaya-Amaya J, Molano-González N, et al. Autoimmune thyroid disease in Colombian patients with systemic lupus erythematosus. Clin Endocrinol (Oxf) 2015;83:943-50

[31] Wohleb ES, Franklin T, Iwata M, et al. Integrating neuroimmune systems in the neurobiology of depression. Nat Rev Neurosci 2016;17:497-511.

[32] Thetford C, Bennett KM, Hodge S, et al. Resilience and vision impairment in older people. J Aging Stud 2015;35:37-48.

[33] Ahern NR, Kiehl EM, Sole M, et al. A review of instruments measuring resilience. Issues Compr Pediatr Nurs 2006;29:103-25.

[34] Tunnicliffe DJ, Singh-Grewal D, Chaitow J, et al. Lupus means sacrifices: perspectives of adolescents and young adults with systemic lupus erythematosus. Arthritis Care Res (Hoboken) 2016;68:828-37.

[35] Azizoddin DR, Zamora-Racaza G, Ormseth SR, et al. Psychological factors that link socioeconomic status to depression/anxiety in patients with systemic lupus erythematosus. J Clin Psychol Med Settings 2017;24:302-15.

[36] Feder A, Nestler EJ, Charney DS. Psychobiology and molecular genetics of resilience. Nat Rev Neurosci 2009;10:446-57.

[37] Resnick B, Klinedinst NJ, Yerges-Armstrong L et al. The impact of genetics on physical resilience and successful aging. J Aging Health 2015;27:1084-104.

[38] Silverman MN, Deuster PA. Biological mechanisms underlying the role of physical fitness in health and resilience. Interface Focus 2014;4:20140040.

[39] Hargardóttir H, van Helvoort HAC, Vonk MC, et al. Exercise in systemic sclerosis intensifies systemic inflammation and oxidative stress. Scand J Rheumatol 2010;39:63-70.

[40] Azar M, Rice DB, Kwakkenbos L, et al. Exercise habits and factors associated with exercise in systemic sclerosis: a Scleroderma Patient-centered Intervention Network (SPIN) cohort study. Disabil Rehabil 2017;39:1-7.

[41] Herrmann M, Schölmerich J, Straub RH. Stress and rheumatic diseases. Rheum Dis Clin North Am 2000;26:737-63.

[42] Lorton D, Lubahn CL, Zautra AJ. Proinflammatory cytokines and sickness behavior in rheumatic diseases. Curr Pharm Des 2008;14:1242-60.

[43] Ostojic P, Zivojinovic S, Reza T, et al. Symptoms of depression and anxiety in Serbian patients with systemic sclerosis: impact of disease severity and socioeconomic factors. Mod Rheumatol 2010;20:353-7.

[44] Graninger M. Behavioral training as additional therapy approach for rheumatoid arthritis. Z Rheumatol 2015;74:579-83. 